\title{
Measurement Validation for ICPS: Matrix Pencil Method for Coriolis Metering with Liquid/Gas Flow
}

\author{
Olga Ibryaeva ${ }^{\mathrm{a}}$, Aleksandr Semenov ${ }^{\mathrm{a}}$ \\ ${ }^{a}$ School of Electrical Engineering and Computer Science \\ South Ural State University \\ Chelyabinsk, Russia \\ ibriaevaol@susu.ru
}

\author{
Manus Henry ${ }^{\mathrm{a}, \mathrm{b}}$ \\ ${ }^{\mathrm{b}}$ Department of Engineering Science \\ University of Oxford \\ Oxford, OX1 3PJ, UK. \\ manus.henry@eng.ox.ac.uk
}

\begin{abstract}
A key requirement for Industrial Cyber-Physical System (ICPS) instrumentation is measurement validation i.e. assessing measurement quality, including detecting and correcting for fault conditions. Coriolis Mass flow meters are used widely throughout industry, but commonly only for singlephase fluids, i.e. either liquids or gases, since accuracy is degraded by gas/liquid flows. The provision of robust measurement in non-ideal conditions is an increasingly important requirement. Here we propose that a modified Matrix Pencil Method is able to track the modes of flowtube vibration excited by gas/liquid flow, and show the results of applying the technique to numerically simulated data.
\end{abstract}

Keywords - Industrial cyber-physical systems, measurement validation, oil and gas, Coriolis mass flow meter, signal processing, matrix pencil method, two-phase flow.

\section{INTRODUCTION}

Autonomic Computing [1, 2] requires systems and components capable of self-configuration, self-optimization, self-healing and self-protection. Applied to sensors, these aspirations align with the concept of the Self-Validating (SEVA) Sensor ([3], a UK National Standard [4]), alongside the broadly equivalent notion of "metrological self-check" developed in Russia [5]. Here, the sensor (taken to include both the transduction and data processing elements) performs self-diagnostics, but further assesses the quality of its measurement data, applying corrections as required for any detected faults, and provides standardized data quality metrics to assist higher level systems evaluate the usefulness of the measurements for particular tasks. If in addition, communication bandwidth constraints require that "the signal will be processed entirely [at] the Point of Acquisition (PoA) [6]" (i.e. within the instrument itself), then the demands placed on instrument capabilities are high indeed.

The Coriolis Mass Flow (CMF) meter, considered by some to be the "almost perfect" flowmeter [7], is widely used in the oil and gas and other industrial sectors, for a wide range of duties including custody transfer. Its “Achilles' heel" has been

This work was conducted with the financial support of the Ministry of Education and Science of the Russian Federation of the Applied Scientific Research «Development of domestic mass Coriolis flow meter for oil and gas industry with the flow measurement function of multiphase flows». Agreement № 14.578.21.0191 from 03.10.2016. Unique identifier of the Applied Scientific Research is RFMEFI57816X0191. an inability to deal with multiphase flows, i.e. mixtures of liquid and gas, which frequently occur in batching applications (for example air + product) or in upstream oil and gas applications (oil and/or water + gas). Significant progress has been made in recent years to improve CMF performance with two- and three-phase mixtures [8], but there is ample scope for further development.

The broader implications for the digital economy of measurement validation are well illustrated by the $\$ 150 \mathrm{~B}$ bunkering (marine fuel) industry [9]. The custody transfer process, taking place between bunker barge and receiving ship, has traditionally been monitored using manual dipping techniques, generating manual transfer receipts. Conventional metering has been unable to deal with the mixtures of heavy fuel oil and air inherent in the bunker transfer. However, the significant delay, inefficiency and fraud potential of manual receipts has driven research to investigate more robust metering techniques. Recently [10], Singapore, the world's largest bunkering port, announced Coriolis metering would be mandatory for all future bunker transfers; the digital economy may now be connected to the key transaction point of the entire industry, via the introduction of robust instrumentation.

In this paper we describe a novel application of a signal processing technique, the Matrix Pencil Method (MPM), to the CMF measurement problem, with the goal of provide a more robust approach to dealing with gas/liquid mixtures. The Coriolis flowtube - the mechanical, vibrating transducer through which passes the fluid to be measured - has multiple modes of vibration, which are readily excited by the mechanical energy of gas/liquid mixtures. These additional modes, alongside the conventional, actively driven mode of vibration, are transferred via motion sensors to the electronic transmitter, which performs the signal processing to extract flow measurement data. Conventional CMF signal processing assumes only one mode of vibration; MPM inherently manages multiple frequency components seamlessly, offering a more robust approach to two-phase signal processing.

The potential benefits can be defined in two stages: firstly, a signal analysis which accommodates multiple modes of vibration can thereby isolate the most important, first, mode from the influence of the others. Secondly, measurement data from the other modes of vibration may themselves prove useful for additional measurement or diagnostic purposes. 
After a survey of prior art, the MPM method is explained. A simulation of CMF sensors signals under two-phase flow conditions is introduced, followed by the results obtained by applying the MPM method. A companion conference paper presents results from obtained applying the MPM method to experimental data for CMF sensor signals obtained under twophase flow conditions.

\section{Coriolis Meter Signal Processing}

CMF signal processing methods for single phase (pure gas or liquid) measurement are well established. The two motion sensor signals are reasonably pure and stable sinusoids, from which the frequency, amplitude and phase are to be derived. The phase difference between the two sensor signals is proportional to mass flow, while the frequency of oscillation is related to the density of the fluid, which is also calculated. Techniques in the recent literature $[11,12]$ include the Fourier Transform, Digital Correlation, Digital Phase Locked Loop, Adaptive Notch Filter, and the Hilbert Transform.

CMF signal processing in two-phase (liquid and gas) flow conditions is challenging $[13,14]$ : all sinusoidal parameters are subject to large and fast variations and must be tracked to high accuracy, and with minimum delay, to provide good control and precise measurements. This may be called the signal processing problem, the subject of the current work. In addition [14], large systematic errors in the mass flow and density measurements are induced; this second issue is beyond the scope of this paper. A range of new CMF signal processing techniques have been developed $[15,16]$, aimed at improving measurement performance in two-phase conditions.

MPM, which was first proposed in [17], is one of the Prony-like methods [18] which estimate the signal as a sum of complex exponentials. Several modifications been proposed since it was first introduced: MPM for undamped sinusoids is thoroughly studied in [19], an improved MPM using low-rank Hankel approximation is suggested in [20], and a quantum version of MPM (where the algorithm is to be run on a quantum computer) is developed in [21].

In our earlier paper [22], MPM is shown to be a promising new approach to CMF signal analysis. The most computationally expensive step of the classical MPM is to calculate the singular value decomposition (SVD) of a matrix composed from the signal samples. Our intention to use MPM on-line over a moving window of data prompted us to develop the Recursive Matrix Pencil Method (RMPM) [23]. When a new point enters the data window, this matrix changes only slightly; it is therefore reasonable to start with the previous SVD and apply a low-rank modification procedure rather than recalculating the SVD in full.

Here, we exploit another aspect of MPM - the ability to readily accommodate two or more modes of vibration, which are likely to occur in two-phase flow. Further, we incorporate an additional modification of the classical MPM to exploit the fact that the two sensors signals have the same frequencies for all vibration modes. We therefore propose Multi-Channel MPM for a more general case of $K$ signals having common poles, although in the problem of CMF signal processing we are dealing initially with the case $K=2$. Note that a similar approach is proposed in [24].

\section{MAtrix PEnCil Method}

\section{A. Classical MPM}

The Matrix Pencil Method is a technique for estimating signal parameters

$$
y(t)=\sum_{k=1}^{M} R_{k} e^{\left(\alpha_{k}+i \omega_{k}\right) t}
$$

from a set of samples

$$
y(n T)=\sum_{k=1}^{M} R_{k} e^{\left(\alpha_{k}+i \omega_{k}\right) n T}=\sum_{k=1}^{M} R_{k} z_{k}^{n}
$$

Here $T$ is the sampling period, $R_{k}=A_{k} e^{i \varphi_{k}}$ are the complex amplitudes, $\alpha_{k}$ are the damping factors, $\omega_{k}=$ $2 \pi f_{k}$ are the frequencies and $z_{k}=e^{\left(\alpha_{k}+i \omega_{k}\right) T}$ are the poles of the signal $y(t)$.

MPM finds estimates for the amplitudes $R_{k}$ and poles $z_{k}$ from the samples $y(n T)=y_{n}, n=0,1, \ldots, N-1$, in two steps. Firstly, it finds the poles $z_{k}$ as the solution of a generalized eigenvalues problem by using the matrix pencil formed from the sampled values $y_{n}$. Secondly, it uses the pole values to estimate $R_{k}$ via a least squares problem.

Define two $(N-L) \times L$ matrices $Y_{1}, Y_{2}$, as follows:

$$
\begin{aligned}
Y_{1} & =\left(\begin{array}{cccc}
y_{L-1} & \cdots & y_{1} & y_{0} \\
y_{L} & \ldots & y_{2} & y_{1} \\
\vdots & \ddots & \vdots & \vdots \\
y_{N-3} & \cdots & y_{N-L} & y_{N-L-1}
\end{array}\right), \\
Y_{2} & =\left(\begin{array}{cccc}
y_{L} & \cdots & y_{2} & y_{1} \\
y_{L+1} & \cdots & y_{3} & y_{2} \\
\vdots & \ddots & \vdots & \vdots \\
y_{N-1} & \cdots & y_{N-L+1} & y_{N-L}
\end{array}\right),
\end{aligned}
$$

where $M \leq L \leq N-M$ is the pencil parameter. It has been shown [17] that $\frac{N}{3}$ and $\frac{2 N}{3}$ are the best choices for $L$ to ensure MPM is least sensitive to noise. In our simulations $L$ has been set as floor (N/3).

In the noise free case, the poles $z_{k}=e^{\left(\alpha_{k}+i \omega_{k}\right) T}$ can be found [17] as the generalized eigenvalues of the matrix pencil $Y_{2}-\lambda Y_{1}$. In other words, the $z_{k}$ are $M$ eigenvalues of $Y_{1}^{\dagger} Y_{2}$, where $\uparrow$ denotes the pseudoinverse or Moore-Penrose inverse. For noisy data, Singular Value Decomposition (SVD) is used to reduce the noise and to estimate the number $M$ of the signal poles. The SVD of the matrix $Y_{1}$ is given by:

$$
Y_{1}=U S V^{T} \text {. }
$$

Here $U$ and $V$ are unitary matrices and $S$ is the diagonal matrix containing the singular values of $Y_{1}$. The superscript $T$ notes the transpose. When arranged by magnitude, the singular values after the first $M$ are typically very close to zero. The order $M$ is thus estimated and the pseudoinverse $Y_{1}^{\dagger}$ is replaced by the rank- $M$ truncated pseudoinverse: 


$$
Y_{1}^{\dagger}=\sum_{m=1}^{M} \frac{1}{\sigma_{m}} v_{m} u_{m}^{T}=V_{0} S_{0}^{-1} U_{0}^{T},
$$

where $\sigma_{1}, \ldots, \sigma_{M}$ are the $M$ largest singular values of the matrix $Y_{1}, v_{m}$ and $u_{m}$ are the corresponding singular vectors, $V_{0}=\left(v_{0}, \ldots, v_{M}\right), U_{0}=\left(u_{0}, \ldots, u_{M}\right), S_{0}=\operatorname{diag}\left(\sigma_{1}, \ldots, \sigma_{M}\right)$.

The estimates of $z_{k}$ can be found ([17]) by computing the eigenvalues of the $M \times M$ nonsymmetrical matrix:

$$
Z_{E}=S_{0}^{-1} U_{0}^{T} Y_{2} V_{0} .
$$

Once $M$ and $z_{k}$ are known, the complex amplitudes $R_{k}$ are then found by solving the following least squares problem:

$$
\left(\begin{array}{c}
y_{0} \\
y_{1} \\
\vdots \\
y_{N-1}
\end{array}\right)=\left(\begin{array}{cccc}
1 & 1 & \cdots & 1 \\
z_{1} & z_{2} & \cdots & z_{M} \\
\vdots & \vdots & \ddots & \vdots \\
z_{1}^{N-1} & z_{2}^{N-1} & \cdots & z_{M}^{N-1}
\end{array}\right)\left(\begin{array}{c}
R_{1} \\
R_{2} \\
\vdots \\
R_{M}
\end{array}\right)
$$

We have summarized classical MPM as it was first presented in [17]. In the next section we describe a modification [25] better suited to the CMF application.

\section{B. Multi-Channel MPM}

Consider a set of $K$ signals $y_{1}(t), y_{2}(t), \ldots, y_{K}(t)$ having the same poles $z_{1}, z_{2}, \ldots, z_{M}$, but, generally speaking, different complex amplitudes $R_{1 k}, R_{2 k}, \ldots, R_{M k}, k=1, \ldots, K$.

Write the matrices $Y_{a k}, Y_{b k}$ which correspond to the signals $y_{k}(t), k=1, \ldots, K$.

$$
\begin{aligned}
Y_{a k}= & \left(\begin{array}{cccc}
y_{k}(L-1) & \ldots & y_{k}(1) & y_{k}(0) \\
y_{k}(L) & \ldots & y_{k}(2) & y_{k}(1) \\
\vdots & \ddots & \vdots & \vdots \\
y_{k}(N-2) & \ldots & y_{k}(N-L) & y_{k}(N-L-1)
\end{array}\right),(9) \\
Y_{b k}= & \left(\begin{array}{cccc}
y_{k}(L) & \ldots & y_{k}(2) & y_{k}(1) \\
y_{k}(L+1) & \ldots & y_{k}(3) & y_{k}(2) \\
\vdots & \ddots & \vdots & \vdots \\
y_{k}(N-1) & \ldots & y_{k}(N-L+1) & y_{k}(N-L)
\end{array}\right) .(10)
\end{aligned}
$$

Each of these matrices admits a factorization:

$$
Y_{a k}=Z_{L} R^{(k)} Z_{R}, \quad Y_{b k}=Z_{L} R^{(k)} Z Z_{R}
$$

Here

$$
\begin{gathered}
Z_{L}=\left(\begin{array}{cccc}
1 & 1 & \ldots & 1 \\
z_{1} & z_{2} & \ldots & z_{M} \\
\vdots & \ddots & \vdots & \vdots \\
z_{1}^{N-L-1} & z_{2}^{N-L-1} & \ldots & z_{M}^{N-L-1}
\end{array}\right) \\
Z_{R}=\left(\begin{array}{cccc}
z_{1}^{L-1} & z_{1}^{L-2} & \ldots & 1 \\
z_{2}^{L-1} & z_{2}^{L-2} & \ldots & 1 \\
\vdots & \ddots & \vdots & \vdots \\
z_{M}^{L-1} & z_{M}^{L-2} & \ldots & 1
\end{array}\right) \\
R^{(k)}=\operatorname{diag}\left[R_{1 k}, R_{2 k}, \ldots, R_{M k}\right], \quad Z=\operatorname{diag}\left[z_{1}, z_{2}, \ldots, z_{M}\right]
\end{gathered}
$$

From the matrices $Y_{a k}, Y_{b k}$ form the block extended matrices $Y_{a E}, Y_{b E}$ of sizes $(N-L) K \times L$, as follows:

$$
Y_{a E}=\left(\begin{array}{c}
Y_{a 1} \\
Y_{a 2} \\
\vdots \\
Y_{a K}
\end{array}\right), \quad Y_{b E}=\left(\begin{array}{c}
Y_{b 1} \\
Y_{b 2} \\
\vdots \\
Y_{b K}
\end{array}\right)
$$

It is easy to see that the poles $z_{k}$ are generalized eigenvalues of the matrix pencil $Y_{b E}-\lambda Y_{a E}$.

$$
Y_{b E}-\lambda Y_{a E}=\left(\begin{array}{cccc}
Z_{L} & 0 & \ldots & 0 \\
0 & Z_{L} & \ldots & 0 \\
\vdots & \ddots & \vdots & \vdots \\
0 & 0 & \ldots & Z_{L}
\end{array}\right)\left(\begin{array}{c}
R^{(1)} \\
R^{(2)} \\
\vdots \\
R^{(K)}
\end{array}\right)(Z-\lambda E) Z_{R}
$$

Using numerical experiments, we will show that finding the poles $z_{1}, z_{2}, \ldots, z_{M}$ as generalized eigenvalues of the matrix pencil $Y_{b E}-\lambda Y_{a E}$ is more efficient for the CMF application than finding generalized eigenvalues of the matrix pencils $Y_{b k}-\lambda Y_{a k}, k=1, \ldots, K$, with their subsequent averaging.

\section{Two-Phase Flow Signal Processing}

\section{A. Modified Random Walk Model for Two-Phase Flow, incorporating Two Vibration Modes}

Simulation studies are an important first means of evaluating the measurement performance of signal tracking algorithms. For example, the "true" values are known, so the measurement errors can be assessed, the influence of noise can be explored systematically etc. As is shown in our companion paper, under experimental two-phase flow conditions, neither the 'true' instantaneous signal properties or instantaneous flow rates of liquid and gas can be asserted with confidence: only their average values can be calculated.

In order to simulate the sensor signals arising in two-phase flow conditions, a modified random walk model was proposed in [16]. Here, we adapt it and add a second mode as follows:

$$
\begin{gathered}
s_{\text {right }}(n)=\sum_{k=1}^{2} A_{k}(n) \sin \left(\omega_{k}(n) n+\frac{\varphi_{k}(n)}{2}\right)+\sigma_{r} \cdot e_{r}(n), \\
s_{\text {left }}(n)=\sum_{k=1}^{2} A_{k}(n) \sin \left(\omega_{k}(n) n-\frac{\varphi_{k}(n)}{2}\right)+\sigma_{l} \cdot e_{l}(n), \\
A_{k}(n)=\frac{\left(A_{k}^{f}(n)-\min \left(A_{k}^{f}(n)\right)\right)\left(A_{k}^{\max }-A_{k}^{\min }\right)}{\max \left(A_{k}^{f}(n)\right)-\min \left(A_{k}^{f}(n)\right)}+A_{k}^{\text {min }}, \\
\omega_{k}(n)=\frac{\left(\omega_{k}^{f}(n)-\min \left(\omega_{k}^{f}(n)\right)\right)\left(\omega_{k}^{\max }-\omega_{k}^{\min }\right)}{\max \left(\omega_{k}^{f}(n)\right)-\min \left(\omega_{k}^{f}(n)\right)}+\omega_{k}^{\min }, \\
\varphi_{k}(n)=\frac{\left(\varphi_{k}^{f}(n)-\min \left(\varphi_{k}^{f}(n)\right)\right)\left(\varphi_{k}^{\max }-\varphi_{k}^{\min }\right)}{\max \left(\varphi_{k}^{f}(n)\right)-\min \left(\varphi_{k}^{f}(n)\right)}+\varphi_{k}^{\min }, \\
A_{k}^{f}(n)=H_{A}(n) * e_{A_{k}}(n), \\
\omega_{k}^{f}(n)=H_{\omega}(n) * e_{\omega_{k}}(n), \\
\varphi_{k}^{f}(n)=H_{\varphi}(n) * e_{\varphi_{k}}(n) .
\end{gathered}
$$


where $s_{\text {right }}(n)$ and $s_{\text {left }}(n)$ are the simulated sensor signals at sample $n$, with common but time varying amplitudes $A_{k}$, frequencies $\omega_{k}$, phase differences $\varphi_{k}, k=1,2$. In addition, $e_{r}(n)$ and $e_{l}(n)$ are uncorrelated white noise processes, $e_{A_{k}}(n), e_{\omega_{k}}(n)$ and $e_{\varphi_{k}}(n)$ are uniformly distributed random noise signals in the interval $(-1,1), \sigma_{r}, \sigma_{l}$ are gain factors for the input noise. $A_{k}^{\max }, A_{k}^{\min }$ are the upper and lower limits for time-varying amplitudes, and $\omega_{k}^{\max }, \omega_{k}^{\min }, \varphi_{k}^{\max }$ and $\varphi_{k}^{\min }$ are the corresponding limits for frequencies and phase differences, respectively. $H_{A}(n), H_{\omega}(n)$ and $H_{\varphi}(n)$ are low-pass filters to limit the rate of change of parameter values.

The bound-limited amplitudes, frequencies and phase differences values are generated via uniform random noise process and passed through low-pass filters to constrain the rate of change.

\section{B. MPM tracking of two modes of simulated signals}

Table I shows parameter values for MRWM used to simulate the two sensor signals. Figure 1 shows one of these signals and its Fast Fourier Transform. The rapidly changing amplitude is apparent, while in the lower plot there are two peaks corresponding to the main drive mode $(85-100 \mathrm{~Hz})$ and to the second mode $(155-180 \mathrm{~Hz})$ of flowtube vibration.

For the MPM calculations the sampling rate was $2.4 \mathrm{kHz}$ and the window length was 50 samples, corresponding to approximately 2.5 periods of the main mode. Compensation has been applied for the fixed time delay in the MPM algorithm.

Figs. 2-7 show MPM and Multi-Channel MPM tracking performances, as follows. For each mode of vibration, the parameters to be tracked are the frequency, amplitude and (for flow measurement purposes) the phase difference between the two sensor signals. Accordingly, Figures 2-4 show frequency, amplitude and phase difference tracking for the main, driven vibration mode, while Figures 5-7 show the corresponding parameter values for the second, excited vibration mode.

Both MPM methods track changes in the signal parameters reasonably well, even for the second mode which has low amplitude. There are, however, on both modes, occasionally larger errors on the phase difference measurement (e.g. at $\mathrm{t}=$ $0.11 \mathrm{~s}$ on the main mode, and at $\mathrm{t}=0.22 \mathrm{~s}$ on the second mode) that may warrant further investigation and algorithmic modification.

To evaluate each method's performance numerically, Table II shows the Root Mean Squared Error (RMSE), calculated using:

$$
R M S E=\sqrt{\frac{1}{n} \sum_{i=1}^{n}(\hat{Y}(n)-Y(n))^{2}} .
$$

where $Y(n)$ and $\hat{Y}(n)$ are the true and estimated values. The results are shown in Table II for the noise free $(\sigma=0.0 \mathrm{~V})$ and noisy $(\sigma=0.0035 \mathrm{~V})$ cases. Multi-Channel MPM performs better both in both cases, especially for the phase difference calculation.
TABLE I.

PARAMETER VALUES FOR MRWM

\begin{tabular}{l|c}
\hline \hline \multicolumn{1}{c|}{ Parameters } & Values \\
\hline Sampling frequency $\left(f_{s}\right)$ & $2.4 \mathrm{kHz}$ \\
\hline Low-pass filter cut-off & $6 \mathrm{~Hz}$ \\
frequencies $\left(f_{A}^{s}, f_{\omega}^{S}, \omega_{\phi}^{S}\right)$ & $A_{1}^{\min }=0.05 \mathrm{~V}, A_{1}^{\max }=0.3 \mathrm{~V}$ \\
\hline Range for amplitude $A_{1}$ & $A_{2}^{\min }=0.005 \mathrm{~V}, A_{2}^{\max }=0.03 \mathrm{~V}$ \\
\hline Range for amplitude $A_{2}$ & $f_{1}^{\min }=85 \mathrm{~Hz}, f_{1}^{\text {max }}=100 \mathrm{~Hz}$ \\
\hline Range for the first frequency & $f_{1}^{\text {min }}=155 \mathrm{~Hz}, f_{1}^{\max }=180 \mathrm{~Hz}$ \\
\hline Range for the second frequency & $\varphi_{k}^{\min }=0^{\circ}, \varphi_{k}^{\max }=4^{\circ}, k=1,2$. \\
\hline Range for phase differences &
\end{tabular}

TABLE II. QUANTIFIED TRACKING PERFORMANCE

\begin{tabular}{|c|c|c|c|}
\hline \multicolumn{4}{|c|}{$\mathbf{0 . 0}$ V, RMSE (main/second mode) } \\
\hline $\begin{array}{c}\text { Method and } \\
\text { Parameter }\end{array}$ & $\begin{array}{c}\text { Frequency } \\
\text { RMSE (Hz) }\end{array}$ & $\begin{array}{c}\text { Amplitude } \\
\text { RMSE (V) }\end{array}$ & $\begin{array}{c}\text { Phase Difference } \\
\text { RMSE }\left(^{\circ} \text { ) }\right.\end{array}$ \\
\hline $\begin{array}{c}\text { Classical MPM } \\
0.085 / 2.63\end{array}$ & $0.0026 / 0.0018$ & $0.42 / 3.92$ \\
\hline $\begin{array}{c}\text { Multi-Channel } \\
\text { MPM }\end{array}$ & $0.085 / 2.36$ & $0.0025 / 0.0017$ & $0.03 / 0.78$ \\
\hline \multicolumn{3}{|c|}{$\mathbf{0 . 0 0 3 5}$ V, RMSE (main/second mode) } \\
\hline $\begin{array}{c}\text { Method and } \\
\text { Parameter }\end{array}$ & $\begin{array}{c}\text { Frequency } \\
\text { RMSE (Hz) }\end{array}$ & $\begin{array}{c}\text { Amplitude } \\
\text { RMSE (V) }\end{array}$ & $\begin{array}{c}\text { Phase Difference } \\
\text { RMSE }\left({ }^{\circ} \text { ) }\right.\end{array}$ \\
\hline $\begin{array}{c}\text { Classical MPM } \\
\text { Multi-Channel } \\
\text { MPM }\end{array}$ & $0.14 / 2.68$ & $0.003 / 0.002$ & $0.5 / 4.34$ \\
\hline
\end{tabular}
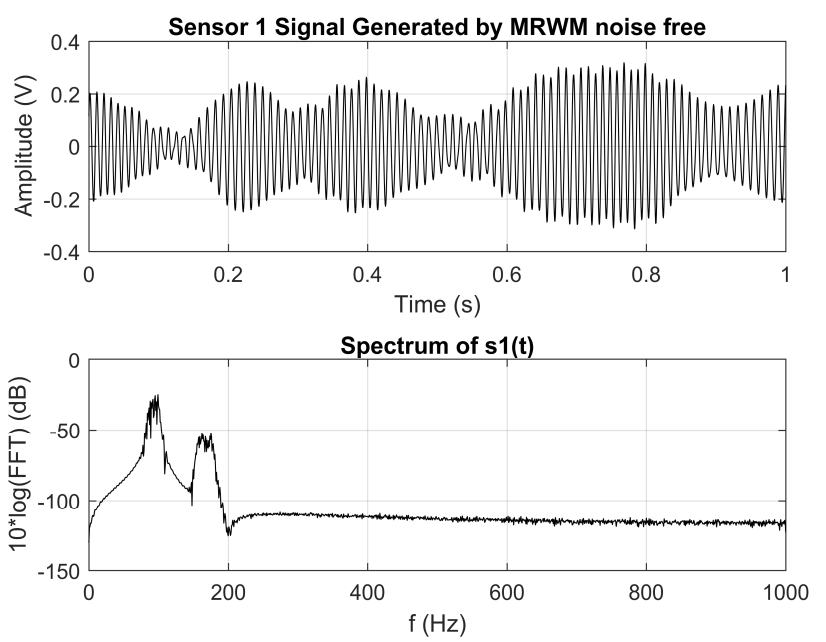

Fig. 1. Simulated CMF meter sensor signal in two-phase flow, incorporating two vibration modes

\section{CONCLUSION}

Industrial Cyber-Physical Systems require robust and sophisticated instrumentation capable of adapting to changing operating conditions, particularly for high value measurements such as custody transfer in the oil and gas industry. The generation of complex data sets, supporting improved diagnostics and measurement validation, is an important means of meeting the measurement challenge of ICPS. 

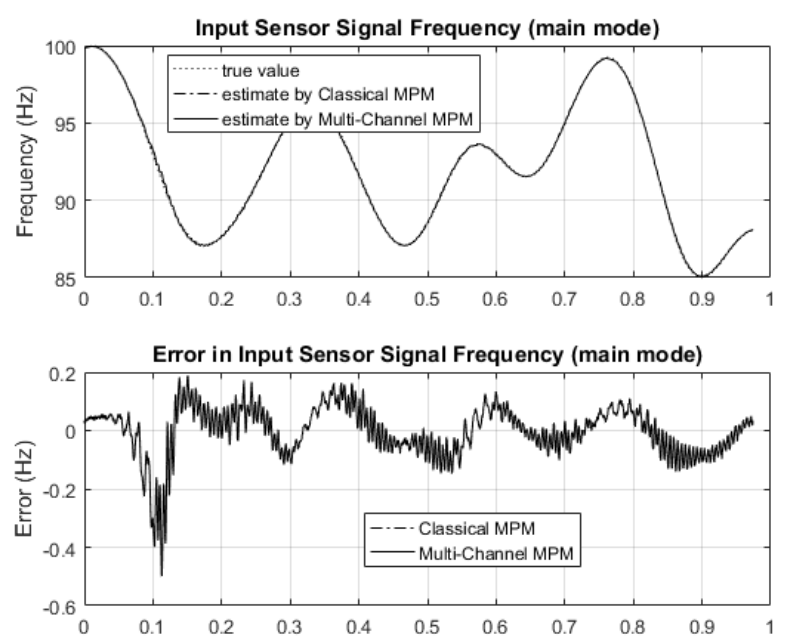

Fig. 2. Main mode, frequency tracking performance
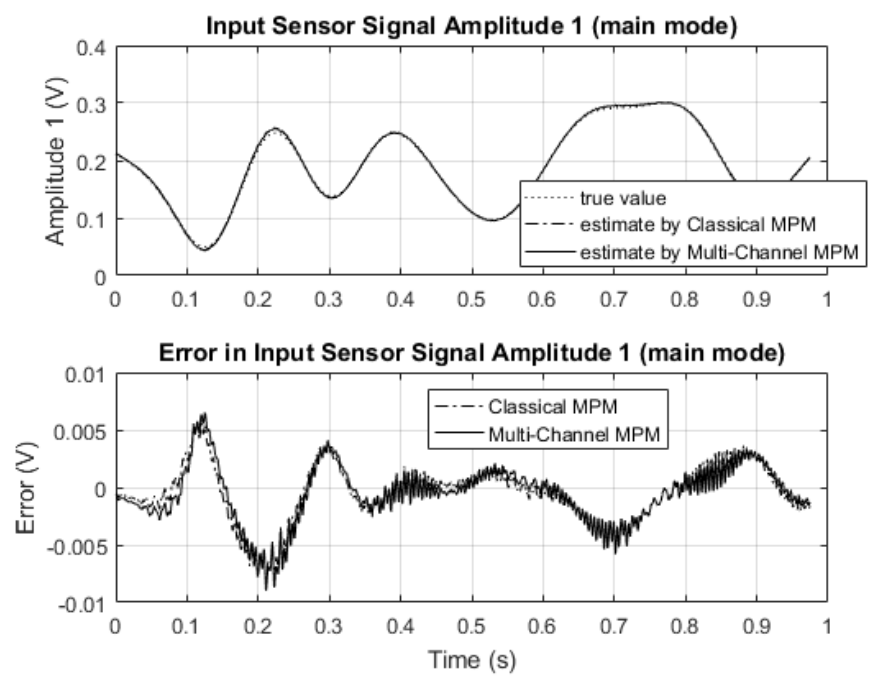

Fig. 3. Main mode, amplitude tracking performance
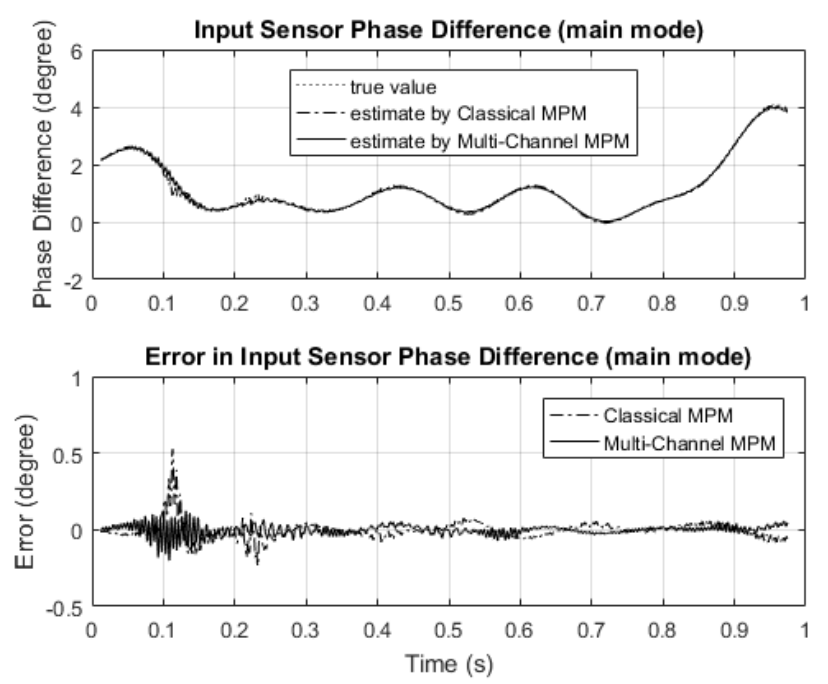

Fig. 4. Main mode, phase difference tracking performance
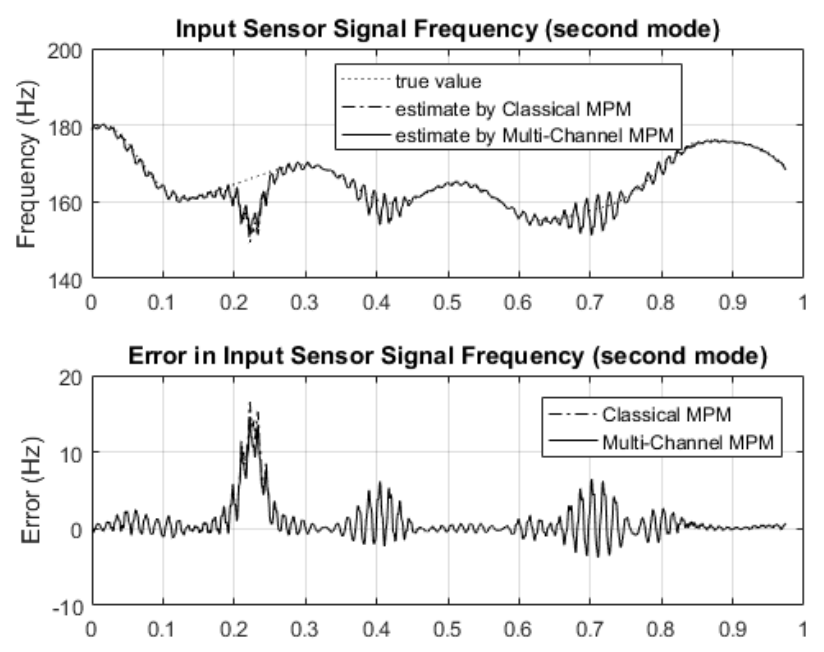

Fig. 5. Second mode, frequency tracking performance
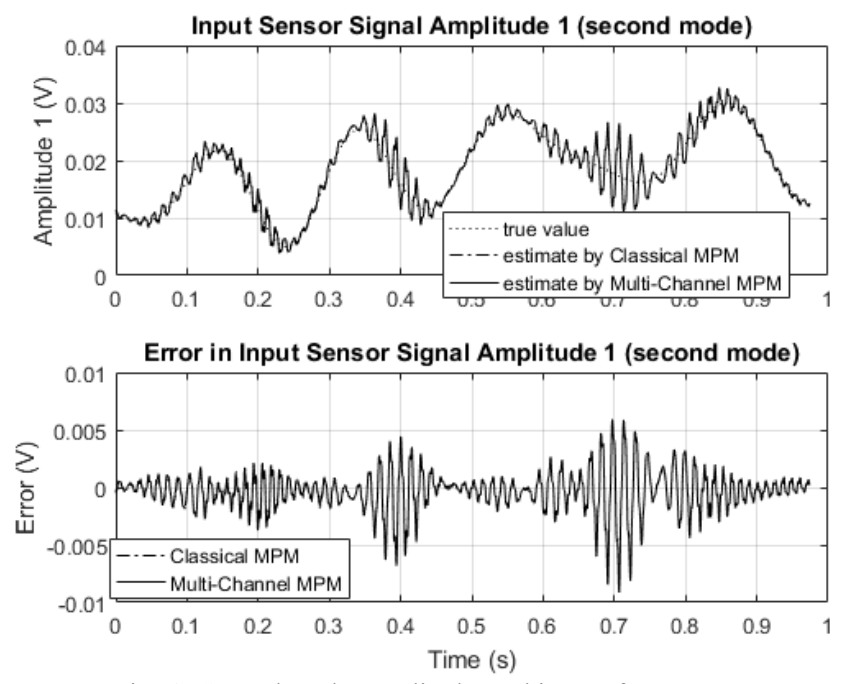

Fig. 6. Second mode, amplitude tracking performance
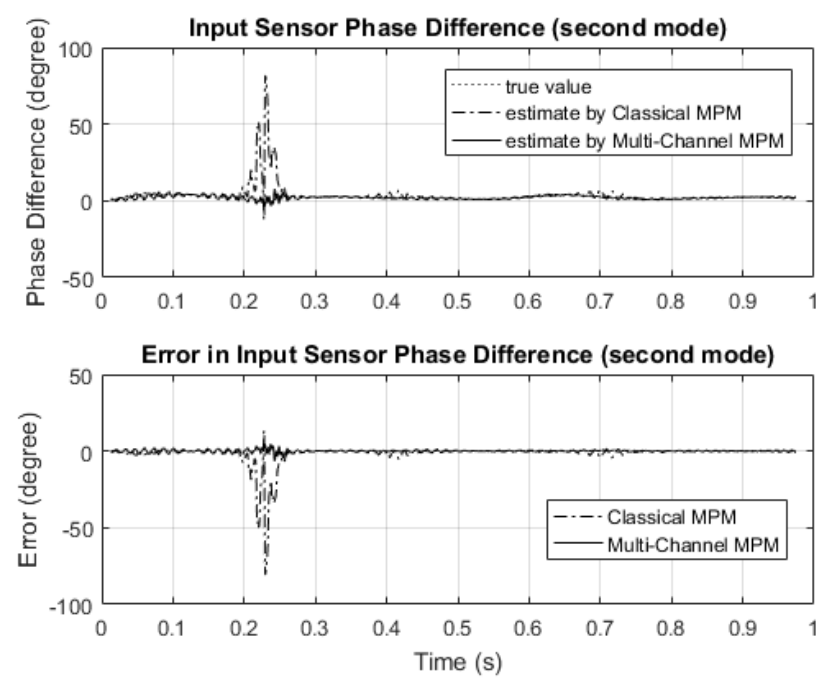

Fig. 7. Second mode, phase difference tracking performance 
In this paper we have described using Multi-Channel Matrix Pencil Method for Coriolis Mass Flow Meter signal processing. The method can track amplitudes, frequencies and phase differences of the main and Coriolis modes. Simulation studies suggest its tracking performance is generally superior to that of the Classical MPM. In our companion paper at this conference, we demonstrate its application to experimental data, where the two modes of vibration are tracked when gas/liquid flow is applied to the flowtube.

Returning to our opening comments, if ICPS systems are to achieve the goals of Autonomic Computing [1, 2]; if the signal processing must occur at the Point of Acquisition; and if measurement validation, including the adaptation to non-ideal conditions such as two-phase flow; then new signal processing techniques are required. These techniques must be capable of a broader range of functionality than of simply delivering the conventional measurement calculation under the assumption of near-ideal conditions. As demonstrated here, MPM, alongside other recent developments such as Prism signal processing $[26,27]$, may form the basis of a flexible toolkit of techniques to enable localized measurement validation. When non-ideal conditions are detected, broader signal processing paths may be seamlessly deployed within the device to extend the useful operating envelope of the instrument, as well as to offer a diagnostic analysis of the current non-ideal conditions.

\section{ACKNOWLEDGEMENT}

The authors would like to express their gratitude to Professor A.L. Shestakov for his continuous encouragement, support and guidance.

\section{REFERENCES}

[1] J. O. Kephart and D. M. Chess, "The vision of autonomic computing", IEEE Computer, vol. 36, no. 1, pp. 41-50, Jan. 2003.

[2] B. Vogel-Heuser, D. Hess, "Guest Editorial - Industry 4.0-Prerequisites and Visions", IEEE Transactions on Automation Science and Engineering, Vol 14, No. 2, April 2016. DOI: 10.1109/TASE.2016.2523639.

[3] M.P. Henry and D.W. Clarke, "The Self-Validating Sensor: Rationale, Definitions and Examples", Control Eng. Pract., vol. 1, pp 585-610, 1993.

[4] BSI, "BS7986:2005, Specification for data quality metrics for industrial measurement and control systems", British Standards Institute, 2005.

[5] R. Taymanov, K. Sapozhnikova, I. Druzhinin, "Sensor Devices with Metrological Self-Check", Sensors \& Transducers Journal, Vol. 10, pp. 30-45, February 2011.

[6] G. Monte, V. Huang, P. Liscovsky, D. Marasco, and A. Agnello,"Standard of things, first step: Understanding and normalizing sensor signals," Industrial Electronics Society, IECON 2013 - 39th Annual Conference of the IEEE, Vienna, 2013, pp. 118-123. DOI: 10.1109/IECON.2013.6699121

[7] R. J. Reizner, "Coriolis - The almost perfect flow meter," Comput. Control Eng. J., vol. 14, no. 4, pp. 28-33.

[8] T. Wang and R. Baker, "Coriolis flowmeters: a review of developments over the past 20 years, and an assessment of the state of the art and likely future directions," Flow Meas. Instrum., vol. 40, pp. 99-123, 2014.
[9] Gregory, D, West, M, Paton, R, Casimiro, R, Boo, S, Low, YK, Henry, MP, Tombs, MS, Duta, MD, Zhou, FB, Zamora, ME, Mercado, R, Machacek, M, Clarke, DW. "Two-Phase Flow Metering using a large Coriolis Mass Flow Meter applied to Ship Fuel Bunkering", Measurement and Control, Sept 2008.

[10] https://www.mpa.gov.sg/web/portal/home/port-of-singapore/circularsand-notices/detail/pc14-08

[11] J. Ruoff, W. Gauchel, and H. Kück, "Advances in Signal Acquisition and Signal Processing of Coriolis Flow Meters," Procedia Eng., vol. 87, pp. 1585-1588, 2014.

[12] P. Yi, Y. Tu, M. Xie, and T. Shen, "Analysis of Phase Difference Tracking Methods for Signal of Coriolis Mass Flowmeter," in 10th World Congress on Intelligent Control and Automation, 2012, pp. 43684373.

[13] M. Tombs, F. Zhou, M. Henry, "Two-Phase Coriolis Mass Flow Metering with High Viscosity Oil", In Press, Flow Measurement and Instrumentation, https://doi.org/10.1016/j.flowmeasinst.2017.11.009.

[14] M. Henry, M. Tombs, M. Duta, F. Zhou, R. Mercado, F. Kenyery, J. Shen, M. Morles, C. Garcia, and R. Langansan, "Two-phase flow metering of heavy oil using a Coriolis mass flow meter: A case study," Flow Meas. Instrum., vol. 17, no. 6, pp. 399-413, 2006.

[15] Y. Tu, H. Yang, H. Zhang, and X. Liu, "CMF Signal Processing Method Based on Feedback Corrected ANF and Hilbert Transformation," Meas. Sci. Rev., vol. 14, no. 1, pp. 41-47, 2014.

[16] M. Li and M. Henry, "Complex Bandpass Filtering for Coriolis Mass Flow Meter Signal Processing," in Industrial Electronics Society (IECON), 2016, pp. 4952-4957.

[17] Y. Hua and T. K. Sarkar, "Matrix Pencil Method for Estimating Parameters of Exponentially Damped/Undamped Sinusoids in Noise," IEEE Trans. Acoust., vol. 38, no. 5, pp. 814-824, 1990.

[18] D. Potts and M. Tasche, "Parameter estimation for nonincreasing exponential sums by Prony - like methods," Linear Algebra Appl., vol. 439, no. 4, pp. 1024-1039, 2013.

[19] J. Enrique, F. del Rio, and T. K. Sarkar, "Comparison between the Matrix Pencil Method and the Fourier Transform Technique for HighResolution Spectral Estimation,” Digit. Signal Process., vol. 6, pp. 108$125,1996$.

[20] B. Lu, D. Wei, B. L. Evans, and A. C. Bovik, "Improved matrix pencil methods," in Conference Record of Thirty-Second Asilomar Conference on Signals, Systems and Computers, 1997, pp. 1433-1437.

[21] A. Steffens, P. Rebentrost, I. Marvian, J. Eisert, and S. Lloyd, "An efficient quantum algorithm for spectral estimation," New J. Phys., vol. 19, pp. 1-14, 2017.

[22] O. Ibryaeva and D. Salov, "Matrix Pencil Method for Coriolis Mass Flow Meter Signal Processing in Two-Phase Flow Conditions," 2017 International Conference on Industrial Engineering (ICIE), 2017, 4 p.

[23] O. Ibryaeva, "Recursive Matrix Pencil Method", 2017 2nd International Ural Conference on Measurements (UralCon) ", 2017, 6 p.

[24] N. Yilmazer, S. Ari, T.K. Sarkar, "Multiple snapshot direct data domain approach and ESPRIT method for direction of arrival estimation", Digital Signal Processing, 18, pp. 561-567, 2008.

[25] M.P. Henry, O.L. Ibryaeva, D.D. Salov, A.S. Semenov, " Matrix Pencil Method for Estimation of Parameters of Vector Processes", Bulletin of the South Ural State University, Series "Mathematical Modelling, Programming and Computer Software", Volume 10, no. 4, pp. 92-104, 2017.

[26] M. Henry, F. Leach, M. Davy, O. Bushuev, M. Tombs, F. Zhou and S. Karout. "The Prism: Efficient Signal Processing for the Internet of Things", IEEE Industrial Electronics Magazine, pp 22 - 32, Dec 2017.

[27] M. Henry. "An Introduction to Prism Signal Processing applied to Sensor Validation", Measurement Techniques, pp 1233 - 1237, Mar 2018. 